\title{
Active Surveillance of Small Renal Masses: A Systematic Review
}

\author{
Elizabeth E. Ellis and Edward Messing* \\ University of Rochester Medical Center, Rochester, NY, USA
}

Received 15 February 2021

Accepted 4 June 2021

Pre-press 19 June 2021

Published 28 August 2021

\begin{abstract}
.
BACKGROUND: Our goal is to review current literature regarding active surveillance (AS) of small renal masses (SRMs) and identify trends in survival outcomes, factors that predict the need for further intervention, and quality of life (QOL).

METHODS: We performed a comprehensive literature search in PubMed and EMBASE and identified 194 articles. A narrative summary was performed in lieu of a meta-analysis due to the heterogeneity of selected studies.

RESULTS: Seventeen articles were chosen to be featured in this review. Growth rate (GR) was not an accurate predictor of malignancy, although it was the characteristic most commonly used to trigger delayed intervention (DI). The mean 5-year overall survival (OS) of all studies was $73.6 \% \pm 1.7 \%$ for AS groups. The combined cancer specific survival (CSS) for AS is $97.1 \% \pm 0.6 \%$, compared to $98.6 \% \pm 0.4 \%$ for the primary intervention (PI) groups, $(p=0.038)$.

CONCLUSIONS: Short and intermediate-term data demonstrate that AS with the option for DI is a management approach whose efficacy (in terms of CSS) approaches that of PI at 5 years, is cost effective, and prevents overtreatment, especially in patients with significant comorbidities.
\end{abstract}

Keywords: Carcinoma, renal cell, biopsy, prognosis, quality of life

\section{INTRODUCTION}

Small renal masses (SRMs) are generally defined as enhancing solid masses $\leq 4 \mathrm{~cm}$ in greatest diameter on cross sectional, contrast enhanced imaging (i.e. computerized tomography (CT) or magnetic resonance imaging (MRI)) [1]. SRMs represent approximately $60 \%$ of all newly identified renal masses [2]. Standard treatment for SRMs currently is primary intervention (PI) which includes minimally invasive or open surgical partial or radical nephrectomy (PN or RN) and thermoablative therapies (TTs) [3]. TTs include percutaneous or surgical cryoablation (CA) and radiofrequency ablation (RFA). Active

${ }^{*}$ Correspondence to: Edward Messing, MD, 601 Elmwood Ave, Box 656, Rochester, NY, 14620, USA. Tel.: 5852753345. E-mail: Edward_Messing@urmc.rochester.edu. surveillance (AS) is a management strategy used for select patients in which tumors are followed by serial imaging [3]. Patients may undergo delayed intervention (DI) based on their preference or as advised due to growth rate (GR) or greatest tumor diameter (GTD) $>4 \mathrm{~cm}$, and/or histology on biopsy [3].

Despite earlier diagnosis in the setting of increased use of cross-sectional imaging for unrelated reasons, mortality rates from renal cell carcinoma (RCC) have not improved [2]. Risks of earlier intervention include overtreatment, as many SRMs are benign [20-30\%], and many RCCs are indolent [1]. There remains a paucity of long-term data and randomized trials to validate current treatment protocols. Recently, however, several groups have published short to intermediate-term results of prospective databases regarding AS. In this review, we seek to compile 
more recent information published since 2010 regarding outcomes of AS, as well as to identify areas where knowledge is lacking. This review will focus on solitary, solid SRMs in adult patients with a normal contralateral kidney. Special considerations for patients with significant comorbidities also will be discussed. Because of length limitations, cystic renal lesions will not be reviewed.

\section{METHODS}

\section{Search strategy and selection}

A comprehensive literature search was performed using the databases PubMed and EMBASE. Articles were queried from the search criteria ([small renal mass] AND [active surveillance] AND [expectant management] AND [renal cell carcinoma]). We also consulted AUA guideline statements [3], CampbellWalsh Urology [4], and other review articles for additional relevant sources.

Articles were reviewed independently by the authors and selected for inclusion based on Cochrane standard methodological procedures [5]. Primary endpoints were tumor GR, overall survival (OS) and cancer specific survival (CSS), time to metastatic disease, time to DI, reliability of biopsy, quality of life (QOL), and cost. Patients' inclusion criteria included all ages with the majority being $\geq 18$ years of age, all races, all genders, all co-morbidities, all life expectancies, all Eastern Cooperative Oncology Group (ECOG) performance statuses, stage T1a renal masses diagnosed by any imaging modality, with or without prior biopsy. Exclusion criteria included non-localized disease, masses $>4 \mathrm{~cm}$, prior treatment for RCC, genetic syndromes predisposing to RCC, and prior systemic therapy for another tumor. All study designs -non-randomized, prospective, and retrospective studies- were included given the paucity of data on this topic. We excluded case reports, other review articles, non-English language manuscripts and papers that were irrelevant to answering our primary end-points.

\section{Bias and statistical analysis}

Likelihood of bias is high due to lack of randomization and the retrospective nature of many of the accepted studies. Due to the heterogeneity of reported data, including different means and frequencies of follow up in the series, and significant differences in selection criteria, a meta-analysis was not feasible.

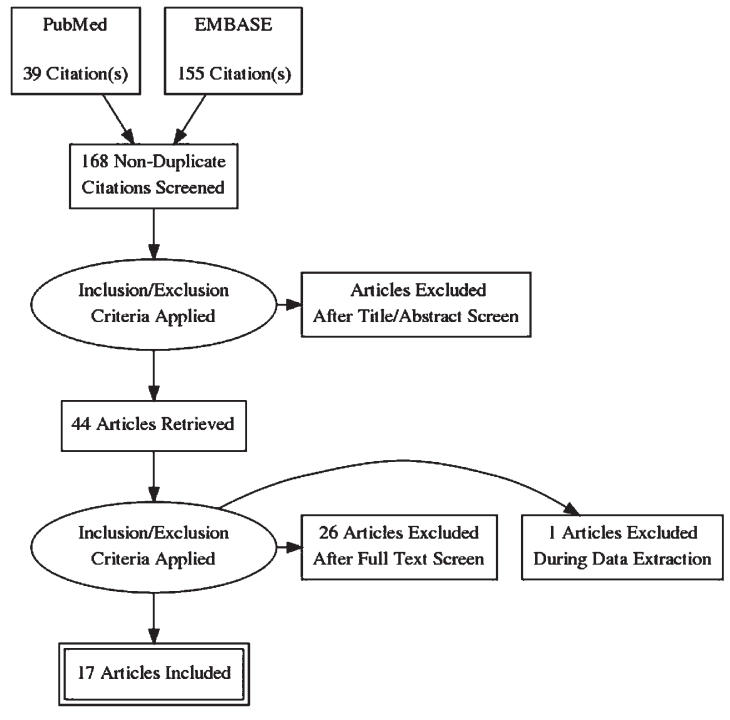

Fig. 1. PRISMA Flow Diagram. 194 Articles were identified from PubMed and EMBASE search engines. EMBASE articles were excluded unless were cited in AUA guidelines or Campbell Walsh Urology and from this a total of 44 articles were carefully assessed. Subsequently, 17 articles were summarized in this discussion.

Instead, a narrative summary of effect estimates was analyzed and reported, and a mean was calculated from pooled data when able. This form of analysis is limited by its failure to account for sizes of the studies relative to each other [6]. Risk of bias was assessed using the Cochrane Risk of Bias Assessment Tool [7].

\section{RESULTS}

\section{Study Selection and quality of the data}

A PRISMA diagram of study selection appears in Fig. 1 [8]. We identified a total of 39 publications from the PubMed search and 155 articles from the EMBASE search engine. Twenty-six articles were duplicates from the PubMed and EMBASE searches. A total of 17 articles were accepted for reporting. Publications were rejected based upon manuscript type: systematic reviews [13], opinion pieces [4], case reports [1]; non-English language manuscripts [2], and inclusion of patients with $>\mathrm{T} 1$ disease [5]. All articles from the EMBASE search that were either not also found in PubMed or cited in AUA guidelines [3] were excluded from the review. Head-to-head comparison was not possible due to the retrospective nature of most of these studies.

To date, there are only two prospective trials regarding survival and GR, and an additional 
prospective trial evaluating QOL while on AS. With the development of multiple AS registries, more light has been shed on the implications of AS; however, data are still limited by the retrospective analysis of prospectively accrued series meaning that the study population is not necessarily powered to analyze specific variables in question. Additional limitations include lack of randomization, strong selection bias for enrolling patients in AS, participation primarily by tertiary referral centers, and lack of long-term data given most publications had a median follow up of approximately 2 years.

Two prospective registries for AS of SRMs have published their data. The Renal Cell Consortium of Canada consisting of seven Canadian academic centers that have used a common surveillance protocol and enrolled a substantial number of patients, though the focus is on older adults with limited life expectancies. At entry into the registry, patients had a renal mass biopsy (RMB) [9]. The second is the Delayed Intervention and Surveillance for Small Renal Masses (DISSRM) consortium. This consists of 3 academic institutions in the United States (Johns Hopkins University, Columbia University and Beth Israel Medical Center in Boston) which has rigorous criteria for follow up and DI, but little information about initial biopsies as only $6.4 \%$ of patients in this registry underwent biopsy initially [10].

\section{Synthesis of results of individual studies}

\section{Imaging modalities}

The AUA guidelines recommend an initial contrast enhanced axial imaging study followed by reimaging with either ultrasound (US) or cross-sectional imaging every 3-6 months in patients who have chosen AS; though they acknowledge that imaging intervals can be customized based on patient and tumor factors [3].When analyzing US imaging one must keep in mind the operator variability of US results; but, if anatomy permits good visualization on US, then US can reliably substitute for CTs or MRIs since its accuracy is similar [10]. In general, cross sectional imaging is performed regularly throughout surveillance. The DISSRM registry uses a strict imaging protocol consisting of reimaging with US after an initial CT or MRI every 4-6 months for 2 years followed by reimaging every $6-12$ months. If US imaging is of poor quality or any change in size is noted, then cross sectional imaging is obtained [10]. This is consistent with findings in a systematic review that evaluated AS surveillance protocols in 20 studies.
CT was the imaging modality used most frequently, and most surveillance imaging was performed biannually, with longer annual intervals for stable SRMs [11]. Several alternative imaging modalities are emerging to evaluate renal masses including contrast enhanced ultrasound imaging (CEUS), which can be used as an alternative to serial cross-sectional imaging. A recent retrospective study of 158 patients undergoing AS noted no difference in median SRM size between CEUS and CT at baseline, 12 months, and 36 months; however, the authors did not directly compare the size differences measured on CEUS and CT between individual SRMs [12].

\section{Tumor size and growth rate}

As a general rule, the larger the SRMs are, the more likely they are to harbor malignancies, with the most common benign lesions being oncocytomas and lipid poor angiomyolipomas (AMLs) (representing about $25 \%$ of SRMs $<1.5 \mathrm{~cm}$ in diameter to $18 \%$ of SRMs $3-4 \mathrm{~cm}$ in diameter) $[13,14]$. Most malignancies are clear cell RCCs of low nuclear grade (nuclear grade 1 and 2), with papillary (usually type I) RCCs and chromophobe carcinomas being the next most common malignancies. Higher grade clear cell and papillary type II RCCs are less common, representing 20-25\% of SRMs in most series [15].

SRMs $<4 \mathrm{~cm}$ in diameter are rarely associated with metastases, and the likelihood of metastases at the time of diagnosis is related to increasing tumor size. Concomitant metastases are almost never found with SRMs $<3 \mathrm{~cm}$ in diameter and virtually "never" with tumors $<2 \mathrm{~cm}$ in diameter $[13,14]$. However, the assumption that all renal cancers that have metastasized and/or progressed locally started off as SRMs drives physicians to offer, and patients to accept immediate PI when a SRM is identified. The information needed to decide whether a patient with a SRM can forgo PI includes frequency of subsequent metastases, and identification of which SRM will spread and when with acceptable accuracy. Further, one should define the characteristics that would trigger DI when a cure, and, as a secondary goal, preservation of renal function, is nearly certain.

Because most SRMs are either benign lesions or indolent malignancies, initially, in patients with comorbidities, advanced age, and/or limited renal reserve, AS has become more widely practiced. For a variety of reasons percutaneous biopsies have not always been performed for patients deemed appropriate for AS, with the thought that periodic monitoring 
for tumor growth, determined by change in tumor diameter or volume, could trigger recommendation for DI, with a secondary trigger being exceeding a certain tumor size (usually $4 \mathrm{~cm}$ GTD) [14].

Because SRMs are not perfect spheres, serial assessments of tumor volume are probably more accurate in quantifying changes in size than measuring only radius or diameter. However, volumetric changes are somewhat challenging to calculate, so usually the GTD is used [13, 16]. A summary of growth rates from all analyzed studies can be found in Table 1.

It is generally thought that larger tumors have a tendency to grow more quickly. In a prospective trial by Petros et al. of 272 patients undergoing AS, a linear mixed-effects model demonstrated that larger tumors size $3-4 \mathrm{~cm}$ grew faster than smaller tumors $<3 \mathrm{~cm}(0.24 \mathrm{~cm} /$ year vs. $0.12 \mathrm{~cm} /$ year respectively, $P<0.001)$ [17]. While the AUA recommends AS as a viable option for SRMs $<3 \mathrm{~cm}$, most studies define progression and recommend DI at a tumor diameter $\geq 4 \mathrm{~cm}$.

There is currently a lack of level 1 evidence to identify patients who should undergo DI after a period of AS, though AUA guidelines recommend, and current practices consist of treatment based on tumor size, GR $>0.5 \mathrm{~cm} / \mathrm{year}$, or patient preference. However, this review did not find much data to support the use of GR to trigger intervention [3].

Rapid growth does not coincide with malignant potential. A prospective multicenter phase 2 "clinical trial" through the Renal Cell Consortium of Canada of 178 patients mean age 73 (median 74), with stage T1a renal masses (mean initial size $2.1 \mathrm{~cm}$ ), deemed unfit for surgery underwent AS after percutaneous needle biopsy [9]. In this study, Jewett et al. demonstrated no difference between GRs of benign and malignant SRMs at a mean follow up of 28 months. The average GR was $0.13 \mathrm{~cm} /$ year for all masses. Furthermore, only some renal masses grew (63\%), and some even decreased in size (26\%) during the follow up period. Subset analysis of the masses that did grow demonstrated an average GR of $0.26 \mathrm{~cm} /$ year. The average GR of biopsy confirmed RCC masses was $0.14 \mathrm{~cm} /$ year compared with benign masses which grew an average of $0.17 \mathrm{~cm} /$ year $(P=0.10)$. Additionally, there was no difference in GR between low grade and high grade RCC SRMs [9]. Similar results were noted in a prospective trial by Siu et al. [18]. In 41 patients undergoing AS with mean follow up of 29 months, mean GR was $0.27 \mathrm{~cm} /$ year with $45 \%$ of tumors having no growth. Histology was available from 17 of the tumors due to DI or biopsy. Of these masses, RCC had a higher GR, however this did not reach statistical significance. Oncocytomas had a mean GR of $0.52 \mathrm{~cm} /$ year, and of the $80 \%$ of confirmed RCCs that grew, the rate was $0.71 \mathrm{~cm} /$ year $(P>0.50)$. One can argue the difference in GRs did not reach significance due to the small sample size; however, one cannot ignore the considerable selection bias, as most of these patients had already been selected either by the surgeon or by patient preference to undergo DI [18].

This is further supported by Uzosike et al. who looked at growth kinetics in 318 patients enrolled in the DISSRM Registry [19]. During the first 6-12 months of observation, tumors grew more slowly but GRs were highly variable. Moreover, they found that for most tumors, growth was non-linear with both more rapid and slower periods of growth over the course of observation. To account for this, they measured both net GR and the maximum interval GR, which is the GR measured between two consecutively obtained imaging studies. There were no external variables associated with increased GR and no statistically significant difference between the GRs of known RCCs and oncocytomas [19]. One can conclude from these current data that GR is neither a reliable predictor of malignancy nor metastatic potential. It has been proposed that this is partially due to the heterogeneity evident in intratumor grading on histology [20]. Moreover, the ability of CT imaging to detect millimeter differences in tumor size, given that the image slice thickness is unlikely to be identical in every scan, further hinders the ability of growth rate to be a reliable surrogate for malignancy.

While GR is not an adequate surrogate for determining malignant potential, the number of positive growth periods may be more predictive of adverse pathology. Jang et al. identified 124 patients who underwent surgery for SRMs (mean initial tumor size $3.4 \mathrm{~cm} \mathrm{SD} \mathrm{2.5)} \mathrm{and} \mathrm{had} \mathrm{multiple} \mathrm{pre-operative}$ imaging studies (median 2 imaging studies) over a mean pre-operative surveillance time of 0.8 year [21]. Patients were divided into two groups based on favorable pathology (defined as benign tumors, chromophobe RCCs, or low grade pT1-2 RCC) and unfavorable pathology (defined as any high grade RCC or locally advanced, low grade pT3-4 RCC); some of these tumors were upstaged after pathologic examination. Growth period was defined as any time between two imaging studies where the difference in GTD was greater than zero. Mean tumor diameter was $2.5 \mathrm{~cm}$ for both favorable and unfavorable groups 
Table 1

Summary of active surveillance for small renal masses growth rate, follow up period and survival data. Risk of bias was calculated using the Cochrane Risk of Bias Assessment Tool

\begin{tabular}{|c|c|c|c|c|c|c|c|c|c|c|c|c|}
\hline References & $\begin{array}{l}\text { Study } \\
\text { Design }\end{array}$ & $\begin{array}{l}\text { Number of } \\
\text { Patients }\end{array}$ & $\begin{array}{l}\text { Mean age, } \\
\text { years } \\
\text { (median) }\end{array}$ & $\begin{array}{l}\text { Median } \\
\text { Follow up } \\
\text { (mean) }\end{array}$ & $\begin{array}{l}\text { Mean tumor } \\
\text { diameter } \\
(\mathrm{cm}) \\
(\text { median })\end{array}$ & $\begin{array}{l}\text { GR all } \\
\text { masses } \\
(\mathrm{cm} / \text { year })\end{array}$ & $\begin{array}{l}\text { \% with } \\
\text { Biopsy }\end{array}$ & $\begin{array}{l}\text { Total } \\
\text { Deaths, } \\
\text { (Deaths due } \\
\text { to RCC) }\end{array}$ & $\begin{array}{l}5 \text { year } \\
\text { Overall } \\
\text { Survival AS } \\
(\mathrm{PI}), p\end{array}$ & $\begin{array}{l}5 \text { year } \\
\text { Cancer } \\
\text { Specific } \\
\text { Survival AS } \\
\text { (PI) } \\
\end{array}$ & $\begin{array}{l}\text { Progression } \\
\text { Free } \\
\text { Survival AS }\end{array}$ & Risk of Bias \\
\hline $\begin{array}{l}\text { Jewett, et al. } \\
\text { [9] }\end{array}$ & $\begin{array}{l}\text { Prospective, } \\
\text { multicenter }\end{array}$ & 178 & $73(74)$ & $(28 \mathrm{~m})$ & 2.1 & 0.13 & \multirow[t]{3}{*}{$56 \%$} & $12(2)$ & & & \multirow[t]{3}{*}{$15.17 \%$} & $\begin{array}{l}\text { Serious risk } \\
\text { of bias }\end{array}$ \\
\hline $\begin{array}{l}\text { Uzosike, et al. } \\
\text { [19] }\end{array}$ & $\begin{array}{l}\text { Prospective, } \\
\text { multicenter }\end{array}$ & 271 & 70.7 & - & $\begin{array}{l}\text { Majority of } \\
\text { tumor had } \\
\text { mean } \leq 2\end{array}$ & 0.05 & & $28(0)$ & & & & $\begin{array}{l}\text { Serious risk } \\
\text { of bias }\end{array}$ \\
\hline $\begin{array}{l}\text { Jang, et al. } \\
{[21]}\end{array}$ & Retrospective & 124 & $(63.5)$ & $\begin{array}{l}0.8 \mathrm{y} \\
\text { favorable } \\
\text { pathology, } \\
0.9 \mathrm{y} \\
\text { unfavorable } \\
\text { pathology }\end{array}$ & $\begin{array}{l}\text { GTD } \\
\text { favorable } \\
\text { pathology } \\
3.2 \text {, } \\
\text { unfavorable } \\
\text { pathology } \\
3.9\end{array}$ & $\begin{array}{l}0.7(1.7) \\
\text { favorable } \\
\text { pathology; } \\
1.6(2.8) \\
\text { unfavorable } \\
\text { pathology }\end{array}$ & & & & & & $\begin{array}{l}\text { Serious risk } \\
\text { of bias }\end{array}$ \\
\hline $\begin{array}{l}\text { Pierorazio, } \\
\text { et al. [10] }\end{array}$ & $\begin{array}{l}\text { Prospective, } \\
\text { multicenter }\end{array}$ & 497 & 70.6 & $2.1 \mathrm{y}$ & 1.9 & 0.11 & \multirow[t]{5}{*}{$6.4 \%$} & 23 (NA) & $\begin{array}{l}75 \%(92) \\
0.06\end{array}$ & $\begin{array}{l}100 \% \\
(99 \%), 0.3\end{array}$ & \multirow[t]{2}{*}{$\begin{array}{l}67 \% \text { at } 5 \\
\text { years }\end{array}$} & $\begin{array}{l}\text { Serious risk } \\
\text { of bias }\end{array}$ \\
\hline $\begin{array}{l}\text { Petros, et al. } \\
\text { [17] }\end{array}$ & $\begin{array}{l}\text { Prospective, } \\
\text { single } \\
\text { institution }\end{array}$ & 272 & 68.5 & $4.8 \mathrm{y}$ & 1.74 & 0.24 & & (4) & & $98 \%$ & & $\begin{array}{l}\text { Serious risk } \\
\text { of bias }\end{array}$ \\
\hline $\begin{array}{l}\text { Cheaib, et al. } \\
\text { [23] }\end{array}$ & $\begin{array}{l}\text { Prospective, } \\
\text { multicenter }\end{array}$ & 785 & & $3.3 \mathrm{y}$ & & & & & $\begin{array}{l}\text { Better } \\
\text { (worse), } \\
<0.001\end{array}$ & $\begin{array}{l}\text { No } \\
\text { difference, } \\
0.6\end{array}$ & \multirow[t]{2}{*}{$\begin{array}{l}62.1 \% \text { at } 7 \\
\text { years }\end{array}$} & $\begin{array}{l}\text { Serious risk } \\
\text { of bias }\end{array}$ \\
\hline $\begin{array}{l}\text { Celtik, et al. } \\
\text { [33] }\end{array}$ & Retrospective & 89 & $(83.4)$ & $39.9 \mathrm{~m}$ & (2.4) & 0.2 & & $16(3)$ & $85.7 \%$ & $95.6 \%$ & & $\begin{array}{l}\text { Serious risk } \\
\text { of bias }\end{array}$ \\
\hline $\begin{array}{l}\text { Tang, et al. } \\
\text { [31] }\end{array}$ & Retrospective & 115 & $(82)$ & $51 \mathrm{~m}$ & (4) & & & $11(2)$ & $58 \%$ & $86 \%$ & $9 \%$ & $\begin{array}{l}\text { Serious risk } \\
\text { of bias }\end{array}$ \\
\hline
\end{tabular}


$(p=0.16)$. They noted a difference in the number of positive growth periods between favorable and unfavorable tumors, with unfavorable tumors being more likely to have at least 2 growth periods during observation compared to the favorable pathology group $(p=0.02)$, while there was no significant difference in GR between the two groups $(p=0.07)$. Furthermore, the unfavorable pathology group had a smaller proportion of zero "positive growth" periods [21]. This can be explained by "immunoediting" in which cancer cells' growth and elimination by immune cells are in a state of equilibrium, until mutations occur that allow cancer cells to enter the escape phase of tumor growth unchecked by the immune system [22].

These factors must be remembered if the decision for DI or delayed biopsy is to be made based on GR, and perhaps one should look to other variables to trigger management changes. Using the number of growth periods as a surrogate should be externally validated and further investigation of variables of tumor biology is warranted. Regardless, of the above findings, tumors that undergo growth should be monitored closely as this could indicate aggressive potential [19].

\section{Survival on AS}

Early outcomes of the DISSRM Registry demonstrated that AS with DI is not an inferior management strategy to PI at a median follow up of 2.1 years (IQR 0.9-3.8). This registry includes patients with SRMs $\leq 4 \mathrm{~cm}$ who chose to undergo AS or PI. Additionally, patients had the option to undergo RMB, but only $6.4 \%$ of patients elected to do so. Patients in the intervention group were significantly younger and had larger tumors compared to the surveillance group (mean age 62.0 years, IQR 24.7-85.3 for PI group vs. 70.6 years, IQR 34.0-93.1 for AS group, $P<0.001$; mean tumor diameter $2.5 \mathrm{~cm}$, IQR $0.09-4.7$ for PI group vs. $1.9 \mathrm{~cm}$, IQR $0.4-7.7$ for AS group). There was no difference in race, gender or BMI between the two groups but there was a difference in performance characteristics (ECOG scores, $P=0.001$ ), Charlson Comorbidity indices $(\mathrm{CCI})(P<0.001)$, cardiovascular index $(\mathrm{CVI})(P=0.002)$, and presence of certain comorbidities including congestive heart failure $(\mathrm{CHF})(P=0.05)$, diabetes mellitus $(P=0.004)$, and hypertension $(P=0.045)$, all of which were greater in those undergoing AS [10].

Intermediate follow up data have supported no difference in OS and CSS between PI and AS groups at 2 years and 5 years $(98 \%$ vs. $96 \%$ OS at 2 years, $92 \%$ vs. $75 \%$ OS at 5 years, $P=0.06 ; 99 \%$ vs. $100 \%$ CSS at 5 years, $P=0.3$, respectively). All-cause mortality for the AS group was also not significantly worse than for the PI group despite worse health status overall (HR 2.2, 95\% CI 1.0-5.0, $P=0.07$ ). This may reflect the short median follow up (2.1 years), though $32 \%$ of patients in the study had follow up $>3$ years. Predictors of mortality included age (HR $1.1,95 \%$ CI 1.04-1.1 $P<0.001)$ and CVI $\geq 2$ on a scale of 0 to $\geq 2$ (HR 3.0, 95\% CI 1.7-13, $P=0.003)(10)$. Longer term data from the DISSRM trial was recently presented at the $21^{\text {st }}$ Annual Meeting of the Society of Urologic Oncology with median follow up of 3.3 years and concluded there was no difference in CSS for AS and PI $(P=0.6)$ but worse OS for AS compared to PI $(P<0.001)$, as expected based on comorbidities [23].

A summary of survival data from all evaluated studies can be found in Table 1. The mean 5-year OS of all studies was $73.6 \% \pm 1.7 \%$ for AS groups. We were unable to perform a pooled statistical analysis on OS for PI groups from our data, however, the cited 5 year OS for PI groups is $92 \%(10)$. This superior OS for the PI group is expected given the selection of patients undergoing AS who generally are older and have more comorbidities. The combined 5- year CSS for AS is significantly worse at $97.1 \% \pm 0.6 \%$, compared to $98.6 \% \pm 0.4 \%$ for the PI groups $(p=0.038)$. However, given the heterogeneity between studies in terms of reported data and means and frequency of follow up, presence of initial biopsy, the role of selection in determining who underwent AS or PI, and indications for DI, it is difficult to interpret this statistically significant difference as being clinically meaningful. Further supporting this interpretation, is that none of the individual reports found a significant difference in CSS between AS and PI groups. Additionally, only a few studies were able to be compared in our statistical analysis due to heterogeneity of reported data.

Progression while on AS in this study was defined as GR $>0.5 \mathrm{~cm} /$ year, GTD $>4.0 \mathrm{~cm}$, development of metastases, or crossover to DI. In the AS group, $67 \%$ of patients "progressed" by 5 years; however, this was because $67 \%$ of tumors either had a high GR $(n=34 / 36)$, had a GTD $>4.0 \mathrm{~cm}(n=2 / 36)$, or crossed over to DI $(n=21 / 36)$, but no patients on AS developed metastases during the follow up period. Indications for DI included patient preference, GR $>0.5 \mathrm{~cm} /$ year or GTD $>4.0 \mathrm{~cm}$. After DI there was no evidence of recurrence at a median follow up of 2.2 years with $15 / 21$ patients having confirmed RCC on final pathology(10). These early 
data suggest that AS with DI is a reasonable option for many patients with SRMs, even malignant ones, although we must take into consideration the paucity of long-term follow up data.

What are the risks of surgery in patients eligible for AS? A multi-institutional study of patients eligible for AS who underwent open partial nephrectomy (PN) for $\leq 4 \mathrm{~cm}$ masses reported outcomes over a median follow up time of 24 to 31 months. Patients were divided into two groups based on risk but not directly compared due to overlap. In one, 55 patients age $>75$ and $\mathrm{CCI}>2$ (though half had CCI $\geq 4$ ) had OS and RFS of $100 \%$ at 2 years, and OS and RFS of $74.7 \%$ and $77.4 \%$, respectively, at 5 years. The second group consisted of 62 patients of any age who had CCI $>4$ and thus were assumed to have a mortality risk $>50 \%$. Compared to the former group, the latter group had worse OS and RFS at 2 years ( $96.7 \%$ and $88.5 \%$, respectively) but had similar OS and RFS at 5 years $(78.1 \%$ and $79.6 \%$, respectively). Patients with CCI $>4$ and high mortality risk are less likely to benefit from partial nephrectomy [24]. Lack of randomized controlled trials precludes definitive judgment about whether AS vs. surgery is better for certain groups; however, it seems surgery is riskier for a select group of patients with significant comorbidities and older age with no evidence of an obvious benefit.

While mortality from treating a SRM is very rare, in general, minimally invasive or open PNs have more complications than TTs, but surgery is more effective oncologically in tumor eradication and metastasis prevention [25]. For PN, significant complications are reported in the 3-8\% range, particularly bleeding and urinary leak (25). When feasible, PN or thermoablation has been recommended over $\mathrm{RN}$, because they preserve more renal tissue (nephron sparing treatment). However, it must be recalled that in the EORTC randomized trial 30904 for solitary renal masses $\leq 5 \mathrm{~cm}$ in diameter considered to be amenable to PN, 531 patients with a "normal" contralateral kidney (based on CT) were randomized to PN or RN. At 9.3 years median follow up, 10-year OS rates of $81.1 \%$ for RN and $75.7 \%$ for PN (HR 1.5 (1.03-2.16, $P=0.03)$ were reported in the intent-to-treat population [26]. Significant differences were no longer seen (HR 1.34, $P=0.17$ ) when only patients with malignancies were analyzed [26]. Despite this, PN patients had significantly better renal function post operatively [27]. The number of deaths attributed to cancer, $4 \mathrm{RN}$ and $8 \mathrm{PN}$ participants, could not account for the differences in OS in the intent-to-treat (or malignancy only) cohorts. While the discrepancy between the intent-to-treat and malignancy-only analyses has been used by some to point out the superiority of nephron sparing surgery, to believe removing the entire kidney, rather than just the tumor for benign disease is somehow protective, defies logic [27]. Additionally, a study by Shuch and colleagues using SEER data indicated that the equal oncologic outcomes and superior OS with PN vs. RN for SRMs may have been due to a higher comorbidity burden and greater age in RN patients [28]. Despite this evidence, currently nephron sparing treatments are recommended and an attempt to have a randomized PN vs. RN study for $\mathrm{T}_{1}$ RCC repeated by the Southwest Oncology Group (SWOG) has not received group or National Cancer Institute approval [3].

One possible explanation for the results of EORTC 30904 is that the negative impact of nephron loss on overall renal function, and its subsequent adverse impact on survival is quite different when the nephron loss is caused by chronic medical conditions (e.g. diabetes, hypertension, peripheral vascular disease, etc.), as opposed to surgical nephron loss [29, 30]. Of course, participants in EORTC 30904 were relatively healthy and believed to have a normal contralateral kidney. They had a median age of 62 , with $63 \%$ having no chronic disease, and nearly $85 \%$ having a World Health Organization performance status of zero [26]. It is possible that the degree of ensuing renal dysfunction of $\mathrm{RN}$ and its impact on cardiovascular disease is not as serious in healthier people as it would be in some of the SRM AS prospective registries, particularly the Renal Cell Consortium of Canada which enrolled patients in part because of many comorbidities and limited life expectancies [9].

A related critical issue in deciding on a course of AS is making an accurate assessment of a patient's longevity were he or she not to have a SRM. CCI and cardiac models have been used for this, and knowing the competing risks of death from conditions other than RCC is critical in deciding on continued monitoring or intervention. Also, it should be remembered that health and comorbidity status are not static, and may not only worsen but occasionally can improve leading to reconsideration for DI [9].

Octogenarians pose an interesting practice dilemma as this age group is more likely to have competing mortality risks, poorer functional status, and increased surgical risk [31]; however tumors have been reported to be more aggressive in patients age 75 and older, presumably in part due to a poor immune response to malignancy [32]. A retrospective study 
of 89 patients age $\geq 80$ (median age 83.4 ) undergoing AS for masses $\leq 7 \mathrm{~cm}$ with a median tumor size $2.4 \mathrm{~cm}$ (IQR $1.7-3.5 \mathrm{~cm}$ ) was conducted. After a median of 39.9 months (IQR 28.1-42.9 months), 9\% of patients underwent DI based on patient preference and tumor GR. Two patients (25\%) in the DI cohort developed metastases. There was no difference in initial size, median R.E.N.A.L (Radius, percent Endo or Exophytic, Nearness of tumor to collecting system or renal sinus, Anterior or posterior location, Location relative to polar lines) nephrometry score, CCI, or Activities of Daily Living Score (ADLs) in patients who developed metastases vs. those who did not. The all-cause mortality was $14.6 \%$, while the cancer specific mortality was $3.4 \%$. The OS and CSS at 5 years was $85.7 \%$ and $95.6 \%$, respectively [33]. This is similar to survival data in all patients undergoing AS suggesting that AS is an adequate management modality in the octogenarian population despite the more aggressive nature of tumors in this age group, especially when considering their poorer surgical outcomes.

\section{Cost and quality of life}

Cost effectiveness and QOL are additional components to consider when deciding on management strategies. Newly diagnosed renal masses cost the healthcare system $\$ 4.4$ billion in 2009 [34]. A Markov model developed by Chang et al. compared costs based on quality adjusted life years (QALY's) of a 65-year-old otherwise healthy patient with an incidentally diagnosed SRM undergoing open or laparoscopic PN vs. percutaneous ablation, AS with DI, and "observation" (presumably AS). They concluded that the predicted lifetime cost per patient of AS alone was \$82,213 2008 USD. However, this model assumed $90 \%$ of patients on AS underwent DI, that $7.25 \%$ of patients developed metastatic disease, and that percutaneous ablation was the method of DI [35]. These estimates are much higher than that cited from current clinical AS series; for example, the metastatic rate in a meta-analysis of $880 \mathrm{AS}$ patients with a mean tumor size of $2.3 \mathrm{~cm}$ was $2.0 \%$ [36]. Because this model is based on questionable data, the cost of AS is likely much lower than that predicted by Chang et al. [34].

However, the cost of AS protocols is significant, not just monetarily, but also because of the burden of missed hours of work due to travel for appointments, cumulative radiation risk, and anxiety due to uncertainty regarding one's disease. AS differs from watchful waiting (WW) in that WW does not require follow-up imaging unless patients develop symptoms [37]. A 2-year prospective trial of 100 patients mean age 72.5 years who were enrolled in WW protocols completed multiple QOL questionnaires at 6, 12 , and 24 months. Patients with increased illness uncertainty had worse QOL physically and psychosocially, and higher distress $(P=0.008, P<0.001$, $P<0.001$ respectively). However, the burden of intrusive thoughts improved from baseline over a period of 24 months, indicating that some psychological aspects of QOL improve over time [38]. Although, this study strictly pertained to patients undergoing WW, and our search did not include any studies that specifically evaluated QOL while on AS, we must consider the psychological burden of illness uncertainty. Indeed, anxiety for those on AS may be greater due to factors such as frequent follow up visits. We should thus offer AS patients access to support groups or other psychological interventions.

\section{Biopsy}

Obtaining a biopsy for SRMs is a topic of controversy. The arguments against initial biopsy are that: 1- all SRMs are presumed to be malignant so that biopsy may not change management. 2- A substantial number of biopsies may be non-diagnostic. 3- Oncocytomas and particularly chromophobe carcinomas may be hard to distinguish from each other, which is made more challenging by tumor heterogeneity. 4Clear cell cancer grading and papillary cancer subtyping can be challenging and not representative due to tumor heterogeneity. 5- Biopsies can alter the radiologic appearance of SRMs particularly if there is biopsy-induced hemorrhage or urine extravasation. 6- Biopsy procedures may theoretically spread (seed) the tumor. And 7- biopsies may be associated with serious procedure-related morbidity. The arguments for performing initial biopsies are that: 1- they are becoming more accurate, with $90 \%$ containing sufficient material for an accurate histologic diagnosis on the first biopsy which increases with a second biopsy [39]. While RMB has a negative predictive value of $70 \%$ [40], it has a $97-99 \%$ and $90-97 \%$ sensitivity and specificity, respectively [40, 41]. 2- Biopsy histology rarely differs from the surgical specimen's histology (including tumor type and grade) with a cited $78 \%$ concordance rate on biopsy in patients who undergo surgery [42]. 3- Serious complications are extremely rare $(<1 \%$ have required an intervention [angioembolization to control bleeding] to manage 
complications in several retrospective series) and are almost always self-limited. And 4- tumor seeding has not been reported in several series [41].

In current practice, biopsy rarely triggers DI in patients on AS. In a study of a prospectively maintained database by Ambani et al. of 118 patients being managed by AS with median follow up of 30 months, 28 patients underwent DI at a median time of 22 months. In the 51 patients who had a biopsy prior to AS enrollment, $37 \%$ were benign, $35 \%$ were malignant, and $28 \%$ were non-diagnostic. They found that the choice to undergo DI was based upon tumor size $>2 \mathrm{~cm}$ compared to size $<2 \mathrm{~cm}(P<0.001)$ regardless of whether a patient underwent biopsy $(P=0.080)$, though the influence of biopsy histology on the decision to undergo DI approached statistical significance $(60 \%$ of patients who underwent DI had confirmed malignancy, while only $25 \%$ of patients in the continued AS group had a confirmed malignancy, $P=0.065$ ). There was no difference in age, performance status, RENAL nephrometry scores, and comorbidities in the DI group vs. those who stayed on AS. Of note, prior to study enrollment, 181 patients underwent initial biopsy and based on these results 130 patients elected to undergo immediate surgery (and were not included in this cohort) and 51 patients chose AS. Factors that were predictive of undergoing DI included size $>2 \mathrm{~cm}$ (HR 3.65, 95\% CI $1.28-10.38, P=0.015)$ and GR (HR 1.26, 95\% CI $1.12-1.41, P<0.001)$, where RMB was not predictive (HR 1.52, 95\% CI 0.70-3.30, $P=0.29$ ). Based on these practice patterns there is an uncertain role for biopsy once a patient is already undergoing AS. Realistically, patient preference is probably the most common reason for undergoing DI, and in this study, patient and surgeon preference was cited as a reason for undergoing DI in $>36 \%$ despite not meeting growth criteria [42]. The strong influence of patient and surgeon preference in choosing to undergo DI is supported in other series with patient preference cited as the reason for DI in $11-71 \%$ and physician preference was cited in up to $41 \%$. GR or tumor size was cited as the deciding factor in in $36-89 \%$ of patients $(9,43-47)$.

One reason that biopsy is not currently used to change management while on AS is the large number of urologists who would not routinely recommend biopsy, despite EAU practice guidelines [37]. In an interview-based study, 73 patients diagnosed with SRMs and 59 board-certified urologists of various subspecialties were given theoretical scenarios and asked their opinions regarding biopsy. Of the urologists, the majority of whom were affiliated with academic centers, $40 \%$ opposed biopsy. Reasons for opposition included diagnostic inaccuracy in 68\%, complication risk in $18 \%$, and potential for tumor seeding in $6 \%$. Urologists did say they would consider biopsy if it had an accuracy rate of $80-95 \%$. After appropriate counseling, 26-29\% of patients in the surgery and AS groups declined biopsy but reasoning was different in each group of patients. Patients undergoing surgery were primarily concerned with missing a diagnosis of cancer (71\%), while patients undergoing AS were concerned about the complications of biopsy (46\%). While almost half the urologists in this group were hesitant to recommend biopsy for a $4 \mathrm{~cm}$ enhancing renal mass in a 60 year old patient, the data show more urologists are recommending biopsy compared to older data from a global survey published in 2012, which demonstrated that $73 \%$ of urologists rarely recommended a biopsy [48].

Biopsies may be beneficial in risk stratifying patients who would benefit from early intervention, and better predict those who would do well on surveillance. In a retrospective single institution study of 380 SRMs that were surgically resected, pathologic features were compared to initial tumor size and survival data with a median follow up of 65 months (IQR 34-87). Of note, $92.9 \%$ of the masses were pT1a, while $7.1 \%$ were upstaged to pT3 at the time of resection. Adverse pathologic features (APFs) were defined as having high nuclear grade, lymphovascular invasion, necrosis, sarcomatoid or rhabdoid features, papillary type II histology, and perinephric fat invasion. The authors found a positive association between size $>3 \mathrm{~cm}$ and the number of APFs. However, there was no difference in disease specific survival based on size alone. When taking into account APFs, disease specific survival was significantly worse in patients who had $\geq 2$ APFs compared to those who had $\leq 1$ APF $(P<0.002)$. Of note, OS and DSS for the entire cohort were similar to other published literature [49]. This suggests that while size alone is not helpful in risk stratifying patients, pathologic evaluation may be useful.

Furthermore, in an analysis of the SEER database, confirmed RCC masses were subdivided based on size in $1 \mathrm{~cm}$ increments and based on histology clear cell, papillary, and chromophobe RCC [15]. The rate of metastatic disease at presentation was calculated for each sub-group. They determined that both size and histology were valuable in predicting metastatic risk. With a cutoff of $3 \%$ risk of metastatic disease, clear cell and papillary RCC should undergo 
intervention at $4 \mathrm{~cm}$, but chromophobe $\mathrm{RCC}$ is more indolent in nature and the risk of metastatic disease is not significant until the mass reaches upwards of $7 \mathrm{~cm}$ [15]. Again, this demonstrates how biopsy would be beneficial in more appropriately risk stratifying patients and preventing overtreatment.

Biopsy utilization is increasing. In a retrospective study of cT1a RCCs using the National Cancer Database by Patel et al., the biopsy rate increased from $8 \%$ in 2004 to $15.3 \%$ in 2015 . Factors associated with undergoing biopsy were increased age, $\mathrm{CCI} \geq 3$, and lower income. Biopsy was found to decrease the rate of patients undergoing PI. Excluding patients with benign disease in this analysis, $36.8 \%$ of patients who had a biopsy underwent non-surgical management, while $11.4 \%$ without a biopsy underwent non-surgical management $(P<0.001)$ [50]. This would have been an even larger difference if patients with benign histology were included in the analysis.

Current studies including a randomized controlled trial by SWOG are ongoing to further elucidate whether a biopsy will impact management. It is clear from numerous studies, that while the role of RMB is uncertain, more information is needed to understand the biology of renal masses and thus to better predict behavior. With no clear surrogates for metastatic potential, we should utilize biopsy more frequently to both decrease the number of patients undergoing PI for benign masses and identify patients who would otherwise undergo AS who would benefit from earlier surgery. The problem is that metastases are rare in SRMs, in general, and particularly in AS series; thus, more biopsies would be needed to demonstrate improved outcomes in a small number of patients, which may not be cost effective. Based on the increased frequency in diagnosis of renal masses with abdominal imaging for unrelated reasons without having improvement in survival outcomes, it is evident that we are over-treating some renal masses just as we initially did with low-risk prostate cancers. Utilizing biopsy for all SRMs prior to treatment selection would help prevent over-treatment and appropriately select patients for AS.

\section{Prognostic models}

Limited by serial imaging alone, it is difficult to predict who will do well on AS. Some prognostic models have been developed to assist with this task. First, the DISSRM score was validated from the DISSRM Registry of 751 patients, $55 \%$ of whom underwent AS. The authors used univariable and multivariable analyses to identify variables that predicted selection of AS. They found that age (age 60-70 administered 1 point, age $>70$ administered 2 points), tumor diameter ( $2-3 \mathrm{~cm}$ administered 1 point, $>3 \mathrm{~cm}$ administered 2 points), CCI ( 1 administered 1 point, $\geq 2$ administered 2 points), and physical component scores from the Medicare Health Outcomes Survey ( $>45$ administered 1 point) were the most predictive of undergoing AS; thus, a score adding all these factors was calculated. Scores ranged from 0-7 with higher scores being more predictive of undergoing AS. Scores $\geq 6$ were predictive of worse OS with HR $10.45(1.25-87.49, P=0.03)$. The authors suggest that scores of 2 or less would be ideal for PI, and scores of 5-7 may be ideal candidates for AS. Patients in the gray zone with scores of 3-4 may benefit from additional workup to identify the correct treatment strategy [51]. Perhaps the impact of the score would be greater if fewer points were allocated to larger tumors and more to smaller ones. While this is ideal for selecting sicker patients for AS over surgery, it does not offer solutions to the dilemma of overtreatment for benign masses or indolent RCCs.

While the prior model offers insight about who might not benefit from PI (primarily because of age and comorbidities), another study helped distinguish variables predictive of developing metastases from SRMs. The current reported metastatic rate of SRMs is approximately $5 \%$, however this is largely dependent on tumor size [52]. In a single institutional retrospective study of 565 patients with median age 55 years (IQR 51-75 years) and median tumor size $2.7 \mathrm{~cm}$ (IQR 2.1-3.4), 2.7\% had metastasis at diagnosis. 549 patients underwent PI and after a median follow up of the entire cohort of 62.8 months (IQR 36.2-92.6), multivariate analysis was used to identify factors that were independently predictive of metastases. These include age $>65$ (HR 5.26, 95\% CI $1.73-16.05, P=0.004)$, ipsilateral synchronous tumors (HR 11.28, 95\% CI 1.95-27.18, $P=0.017$ ), symptomatic tumors (HR 4.63, 95\% CI 1.56-10.92, $P=0.032$ ), and size $>3 \mathrm{~cm}$ (HR 6.83, 95\% CI $1.72-22.62, P=0.006)$. When adding a weighted sum of these risk factors ( 2 points allocated to ipsilateral synchronous tumors and 1 point for the other factors), patients with $\geq 2$ points had increased risk of metastases (HR 12.56, 95\% CI 5.52-22.85) with $81.3 \%$ sensitivity, and $73.4 \%$ specificity [53]. Based on this study, age $>65$, presence of ipsilateral tumors, symptomatic tumors, and size $>3 \mathrm{~cm}$ are factors to consider in patients who would be better stratified to undergo PI. 
A recent quality improvement analysis by the MUSIC-KIDNEY Collaborative sought to identify cases in which surgery could have been avoided. Developing a database from centers across the state of Michigan, they identified 74 cT1 benign renal masses on final pathology after surgical intervention. They concluded that if other management strategies were employed, surgery could have been avoided in a significant number of these cases. The authors suggest strategies to prevent surgery for non-malignant pathology include RMB, AS, and greater use of repeat imaging [54].

\section{DISCUSSION}

So, what should a urologist do with a patient with a SRM who has a negative metastatic work up? A thorough assessment of comorbidities with an accurate estimation of remaining years of life must be made. Patients with a limited life expectancy, particularly if renal function is expected to worsen after intervention would certainly point one to recommend AS. However, even in healthy elderly individuals, it is hard to prove that active treatment for masses up to $7 \mathrm{~cm}$ in diameter $\left(\mathrm{T}_{1 \mathrm{a}}\right.$ and $\left.\mathrm{T}_{1 \mathrm{~b}}\right)$ prolongs survival [55]. While this conclusion was based on a retrospective single institution (Cleveland Clinic) series with obvious selection biases characteristic of clinical series, management between PI and AS in multivariable analyses did not influence mortality $(P=0.67)$, while male gender, tumor size and advancing age did [55]. Similar results of AS vs. PI $(P=0.6)$ have been presented by the DISSRM consortium [23].

All that said, for healthy patients with a normal contralateral kidney we advise that a RMB be performed (and repeated if non-diagnostic) and AS be recommended for all patients with benign asymptomatic lesions. AS and PI should both be discussed as options for Grade 1 or 2 clear cell, papillary type I and chromophobe carcinomas. If grade 3 or 4 clear cell or papillary type 2 carcinomas are diagnosed, PI should be recommended.

What intervention should be chosen? Tumor location, a major component of the RENAL nephrometry score predicts complexity of PN, but also impacts feasibility and success of TTs [56]. A hilar location often makes TTs ineffective because of the "heat sink" effect of brisk blood flow through major renal vessels which prevents achieving the extremes in temperature needed for RFA or CA to kill treated tissue [13, 57]. Other systems evaluating complexity of surgery are referenced in Sun et al. including the PADUA score defined by Ficarra, et al. and could be used for similar reasons $[58,59]$.

If a patient with an indolent malignancy on RMB accepts AS, a rigorous schedule of intense imaging is needed to assess growth kinetics. In general metastases are not associated with tumors that are stable in size or regress (about 30\% in most series) [14]. GRs $<2 \mathrm{~mm} /$ year should encourage continued AS. While the data summarized in this review refute that GR alone is indicative of risk for metastatic disease, a GR $>2.5-5.0 \mathrm{~mm} /$ year has previously been shown to be associated with ultimately developing metastases and should provoke biopsy, if one had not been performed initially [44]. Similarly, urologists should recommend biopsy or DI for GTD beyond 4 $\mathrm{cm}$ and multiple positive growth periods, i.e. persistent growth between a series of follow up imaging $[23,44]$.

Limitations to the data presented include strong selection bias and lack of randomized trials. Further investigation is needed regarding long term outcomes and the generalizability of the data to community practice. Other predictors of more aggressive disease such as growth periods, APFs and other biologic markers should be further investigated to better select candidates for AS.

\section{CONCLUSIONS}

Short and intermediate-term data demonstrate that AS with the option for DI is a safe treatment modality with almost equal survival outcomes to PI groups at 2 and 5 years, is cost effective, and prevents overtreatment, especially in patients with significant comorbidities. Surgery and TT may have adverse consequences, so we should avoid over treatment in not only our elderly population with significant comorbidities but also consider AS in patients who are young and otherwise healthy. While GR alone is not beneficial in predicting malignancy, prognostic models, growth periods, and histologic data from biopsies may be beneficial in determining patients who should undergo DI. Routine RMBs may be beneficial in further estimating risk and preventing over treatment. Further research is needed regarding longterm follow up data and QOL and cost analysis of AS.

\section{ACKNOWLEDGMENTS}

The authors have no acknowledgments. 


\section{FUNDING}

The authors report no funding.

\section{AUTHOR CONTRIBUTIONS}

Elizabeth Ellis and Edward Messing both reviewed and selected the articles and analyzed the data. Elizabeth Ellis extracted data and drafted the manuscript which was critically reviewed by Edward Messing. Both authors agree to be accountable for the accuracy and integrity of the work.

\section{CONFLICT OF INTEREST}

The authors have no conflict of interest to report.

\section{REFERENCES}

[1] Akdogan B, Gudeloglu A, Inci K, Gunay LM, Koni A, Ozen H. Prevalence and Predictors of Benign Lesions in Renal Masses Smaller Than $7 \mathrm{~cm}$ Presumed to be Renal Cell Carcinoma. Clinical Genitourinary Cancer. 2012;10(2):121-5.

[2] Marra G, Oderda M, Allasia M, Munegato S, Joniau S, Gontero P. A Review on the Management of Small Renal Masses: Active Surveillance Versus Surgery. Anticancer Agents Med Chem. 2018;18(7):940-50.

[3] Campbell S, Uzzo RG, Allaf ME, Bass EB, Cadeddu JA, Chang A, et al. Renal Mass and Localized Renal Cancer: AUA Guideline. J Urol. 2017;198(3):520-9.

[4] Steven C. Campbell MD P, Brian R. Lane MD, PhD and Phillip M. Pierorazio MD. Malignant Renal Tumors. In: Alan W. Partin MD P, Roger R. Dmochowski MD, MMHC, FACS, Louis R. Kavoussi MD, MBA and Craig A. Peters MD, editor. Campbell-Walsh-Wein Urology, Twelfth Edition. Twelfth Edition ed. Philadelphia, PA: Elsevier, Inc.; 2021. pp. 2133-84.e15.

[5] Higgins JPT TJ, Chandler J, Cumpston M, Li T, Page MJ, Welch VA (editors). Cochrane Handbook for Systematic Reviews of Interventions version 6.1 [updated updated September 2020. Available from: www.training.cochrane. org/handbook.

[6] McKenzie JE BS. Synthesizing and presenting findings using other methods. 2021. In: Cocrane Handbook for Systematic Reviews of Interventions version 62 [Internet]. Cochrane. Available from: Available from www.training. cochrane.org/handbook.

[7] Sterne JAC HM, Reeves BC, Savović J, Berkman ND, Viswanathan M, Henry D, Altman DG, Ansari MT, Boutron I, Carpenter JR, Chan AW, Churchill R, Deeks JJ, Hróbjartsson A, Kirkham J, Jüni P, Loke YK, Pigott TD, Ramsay CR, Regidor D, Rothstein HR, Sandhu L, Santaguida PL, Schünemann HJ, Shea B, Shrier I, Tugwell P, Turner L, Valentine JC, Waddington H, Waters E, Wells GA, Whiting PF, Higgins JPT. ROBINS-I: a tool for assessing risk of bias in non-randomized studies of interventions. BMJ. 2016;355(i4919).

[8] Moher D, Shamseer L, Clarke M, Ghersi D, Liberati A, Petticrew M, et al. Preferred reporting items for systematic review and meta-analysis protocols (PRISMA-P) 2015 statement. Systematic Reviews. 2015;4(1):1.

[9] Jewett MA, Mattar K, Basiuk J, Morash CG, Pautler SE, Siemens DR, et al. Active surveillance of small renal masses: progression patterns of early stage kidney cancer. Eur Urol. 2011;60(1):39-44.

[10] Pierorazio PM, Johnson MH, Ball MW, Gorin MA, Trock BJ, Chang P, et al. Five-year analysis of a multi-institutional prospective clinical trial of delayed intervention and surveillance for small renal masses: the DISSRM registry. Eur Urol. 2015;68(3):408-15.

[11] Rebez G, Pavan N, Mir MC. Available active surveillance follow-up protocols for small renal mass: a systematic review. World J Urol. 2021.

[12] Bertelli E, Palombella A, Sessa F, Baldi I, Morelli N, Verna $\mathrm{S}$, et al. Contrast-enhanced ultrasound (CEUS) imaging for active surveillance of small renal masses. World J Urol. 2021.

[13] Kunkle DA, Egleston BL, Uzzo RG. Excise, ablate or observe: the small renal mass dilemma-a meta-analysis and review. J Urol. 2008;179(4):1227-33; discussion 33-4.

[14] Jewett MA, Zuniga A. Renal tumor natural history: the rationale and role for active surveillance. Urol Clin North Am. 2008;35(4):627-34; vii.

[15] Daugherty M, Sedaghatpour D, Shapiro O, Vourganti S, Kutikov A, Bratslavsky G. The metastatic potential of renal tumors: Influence of histologic subtypes on definition of small renal masses, risk stratification, and future active surveillance protocols. Urol Oncol. 2017;35(4):153 .e15-.e20.

[16] Nayyar M, Cheng P, Desai B, Cen S, Desai M, Gill I, et al. Active Surveillance of Small Renal Masses: A Review on the Role of Imaging With a Focus on Growth Rate. J Comput Assist Tomogr. 2016;40(4):517-23.

[17] Petros FG, Venkatesan AM, Kaya D, Ng CS, Fellman BM, Karam JA, et al. Conditional survival of patients with small renal masses undergoing active surveillance. BJU Int. 2019;123(3):447-55.

[18] Siu W, Hafez KS, Johnston WK, 3rd, Wolf JS, Jr. Growth rates of renal cell carcinoma and oncocytoma under surveillance are similar. Urol Oncol. 2007;25(2):115-9.

[19] Uzosike AC, Patel HD, Alam R, Schwen ZR, Gupta M, Gorin MA, et al. Growth Kinetics of Small Renal Masses on Active Surveillance: Variability and Results from the DISSRM Registry. J Urol. 2018;199(3):641-8.

[20] Ball MW, Bezerra SM, Gorin MA, Cowan M, Pavlovich $\mathrm{CP}$, Pierorazio PM, et al. Grade heterogeneity in small renal masses: potential implications for renal mass biopsy. J Urol. 2015;193(1):36-40.

[21] Jang A, Patel HD, Riffon M, Gorin MA, Semerjian A, Johnson $\mathrm{MH}$, et al. Multiple growth periods predict unfavourable pathology in patients with small renal masses. BJU Int. 2018;121(5):732-6.

[22] Schreiber RD, Old LJ, Smyth MJ. Cancer immunoediting: integrating immunity's roles in cancer suppression and promotion. Science. 2011;331(6024):1565-70.

[23] Cheaib JG PH, Alam R, et al. editor Active Surveillance versus Primary Intervention for Clinical T1a Kidney Tumors: Ten-Year Experience of the DISSRM Prospective Comparative Study. 21st Annual Meeting of the Society of Urologic Oncology; 2020 December 3, 2020.

[24] Audenet F, Audouin M, Drouin SJ, Comperat E, Mozer P, Chartier-Kastler E, et al. Charlson score as a single pertinent criterion to select candidates for active surveillance 
among patients with small renal masses. World J Urol. 2014; 32(2):513-8.

[25] Klatte T, Shariat SF, Remzi M. Systematic review and metaanalysis of perioperative and oncologic outcomes of laparoscopic cryoablation versus laparoscopic partial nephrectomy for the treatment of small renal tumors. J Urol. 2014; 191(5):1209-17.

[26] Van Poppel H, Da Pozzo L, Albrecht W, Matveev V, Bono A, Borkowski A, et al. A prospective, randomised EORTC intergroup phase 3 study comparing the oncologic outcome of elective nephron-sparing surgery and radical nephrectomy for low-stage renal cell carcinoma. Eur Urol. 2011;59(4):543-52.

[27] Scosyrev E, Messing EM, Sylvester R, Campbell S, Van Poppel H. Renal function after nephron-sparing surgery versus radical nephrectomy: results from EORTC randomized trial 30904. Eur Urol. 2014;65(2):372-7.

[28] Shuch B, Hanley JM, Lai JC, Vourganti S, Setodji CM, Dick $\mathrm{AW}$, et al. Adverse health outcomes associated with surgical management of the small renal mass. J Urol. 2014;191(2): 301-8.

[29] Go AS, Chertow GM, Fan D, McCulloch CE, Hsu CY. Chronic kidney disease and the risks of death, cardiovascular events, and hospitalization. N Engl J Med. 2004;351(13): 1296-305.

[30] Demirjian S, Lane BR, Derweesh IH, Takagi T, Fergany A, Campbell SC. Chronic kidney disease due to surgical removal of nephrons: relative rates of progression and survival. J Urol. 2014;192(4):1057-62.

[31] Tang DH, Nawlo J, Chipollini J, Gilbert SM, Poch M, Pow-Sang JM, et al. Management of Renal Masses in an Octogenarian Cohort: Is There a Right Approach? Clin Genitourin Cancer. 2017;15(6):696-703.

[32] O'Malley RL, Godoy G, Phillips CK, Taneja SS. Is surveillance of small renal masses safe in the elderly? BJU Int. 2010;105(8):1098-101.

[33] Celtik KE, Shah PH, Patel VR, Moreira DM, George $\mathrm{AK}$, Iacovelli $\mathrm{V}$, et al. Active surveillance for incidental renal mass in the octogenarian. World J Urol. 2017;35(7): 1089-94.

[34] Jemal A, Siegel R, Ward E, Hao Y, Xu J, Thun MJ. Cancer statistics, 2009. CA Cancer J Clin. 2009;59(4):225-49.

[35] Chang SL, Cipriano LE, Harshman LC, Garber AM, Chung BI. Cost-effectiveness analysis of nephron sparing options for the management of small renal masses. J Urol. 2011;185(5):1591-7.

[36] Smaldone MC, Kutikov A, Egleston BL, Canter DJ, Viterbo $\mathrm{R}$, Chen DY, et al. Small renal masses progressing to metastases under active surveillance: a systematic review and pooled analysis. Cancer. 2012;118(4):997-1006.

[37] B. Ljungberg LA, K. Bensalah, et al. EAU guidelines on renal cell carcinoma. 2020 [Available from: https://uroweb. org/wp-content/uploads/EAU-Pocket-Guidelines-onRenal-Cell-Carcinoma-2020.pdf

[38] Parker PA, Alba F, Fellman B, Urbauer DL, Li Y, Karam JA, et al. Illness uncertainty and quality of life of patients with small renal tumors undergoing watchful waiting: a 2-year prospective study. Eur Urol. 2013;63(6):1122-7.

[39] Richard PO, Jewett MA, Bhatt JR, Kachura JR, Evans AJ, Zlotta AR, et al. Renal Tumor Biopsy for Small Renal Masses: A Single-center 13-year Experience. Eur Urol. 2015;68(6):1007-13.

[40] Patel HD, Johnson MH, Pierorazio PM, Sozio SM, Sharma R, Iyoha E, et al. Diagnostic Accuracy and Risks of Biopsy in the Diagnosis of a Renal Mass Suspicious for Localized
Renal Cell Carcinoma: Systematic Review of the Literature. J Urol. 2016;195(5):1340-7.

[41] Marconi L, Dabestani S, Lam TB, Hofmann F, Stewart F, Norrie J, et al. Systematic Review and Meta-analysis of Diagnostic Accuracy of Percutaneous Renal Tumour Biopsy. Eur Urol. 2016;69(4):660-73.

[42] Ambani SN, Morgan TM, Montgomery JS, Gadzinski AJ, Jacobs BL, Hawken S, et al. Predictors of Delayed Intervention for Patients on Active Surveillance for Small Renal Masses: Does Renal Mass Biopsy Influence Our Decision? Urology. 2016;98:88-96.

[43] Brunocilla E, Borghesi M, Schiavina R, Della Mora L, Dababneh H, La Manna G, et al. Small renal masses initially managed using active surveillance: results from a retrospective study with long-term follow-up. Clin Genitourin Cancer. 2014;12(3):178-81.

[44] Crispen PL, Viterbo R, Boorjian SA, Greenberg RE, Chen DY, Uzzo RG. Natural history, growth kinetics, and outcomes of untreated clinically localized renal tumors under active surveillance. Cancer. 2009;115(13):2844-52.

[45] Haramis G, Mues AC, Rosales JC, Okhunov Z, Lanzac AP, Badani K, et al. Natural History of Renal Cortical Neoplasms During Active Surveillance With Follow-up Longer Than 5 Years. Urology. 2011;77(4):787-91.

[46] Kouba E, Smith A, McRackan D, Wallen EM, Pruthi RS. Watchful waiting for solid renal masses: insight into the natural history and results of delayed intervention. J Urol. 2007;177(2):466-70; discussion 70.

[47] Rosales JC, Haramis G, Moreno J, Badani K, Benson MC, McKiernan J, et al. Active surveillance for renal cortical neoplasms. J Urol. 2010;183(5):1698-702.

[48] Barwari K, de la Rosette JJ, Laguna MP. The penetration of renal mass biopsy in daily practice: a survey among urologists. J Endourol. 2012;26(6):737-47.

[49] Syed JS, Nawaf CB, Rosoff J, Bryson C, Nguyen KA, Suarez-Sarmiento A, et al. Adverse pathologic characteristics in the small renal mass: implications for active surveillance. Can J Urol. 2017;24(2):8759-64.

[50] Patel HD, Nichols PE, Su ZT, Gupta M, Cheaib JG, Allaf ME, et al. Renal Mass Biopsy is Associated with Reduction in Surgery for Early-Stage Kidney Cancer. Urology. 2020;135:76-81.

[51] Sotimehin AE, Patel HD, Alam R, Gorin MA, Johnson MH, Chang P, et al. Selecting Patients with Small Renal Masses for Active Surveillance: A Domain Based Score from a Prospective Cohort Study. J Urol. 2019;201(5):886-92.

[52] Lughezzani G, Jeldres C, Isbarn H, Perrotte P, Shariat SF, Sun M, et al. Tumor size is a determinant of the rate of stage T1 renal cell cancer synchronous metastasis. J Urol. 2009;182(4):1287-93.

[53] Tan YG, Khalid MFB, Villanueva A, Huang HH, Chen K, Sim ASP. Are small renal masses all the same? Int J Urol. 2020;27(5):439-47.

[54] Peabody H, Patel A, Johnson A, Mirza M, Noyes SL, Schervish E, et al. Development of a Novel Scoring System Quantifies Opportunities to Reduce Surgery for Benign Renal Neoplasms: A Retrospective Quality Improvement Analysis within the MUSIC-KIDNEY Collaborative. J Urol. 2020;204(6):1160-5.

[55] Lane BR, Abouassaly R, Gao T, Weight CJ, Hernandez AV, Larson BT, et al. Active treatment of localized renal tumors may not impact overall survival in patients aged 75 years or older. Cancer. 2010;116(13):3119-26.

[56] Kutikov A, Uzzo RG. The R.E.N.A.L. nephrometry score: a comprehensive standardized system for quantitating renal 
tumor size, location and depth. J Urol. 2009;182(3): 844-53.

[57] Zagoria RJ, Hawkins AD, Clark PE, Hall MC, Matlaga BR, Dyer RB, et al. Percutaneous CT-guided radiofrequency ablation of renal neoplasms: factors influencing success. AJR Am J Roentgenol. 2004;183(1):201-7.

[58] Sun M, Vetterlein M, Harshman LC, Chang SL, Choueiri TK, Trinh QD. Risk Assessment in Small Renal Masses: A Review Article. Urol Clin North Am. 2017;44(2):189-202.
[59] Ficarra V, Novara G, Secco S, Macchi V, Porzionato A, De Caro R, et al. Preoperative aspects and dimensions used for an anatomical (PADUA) classification of renal tumours in patients who are candidates for nephron-sparing surgery. Eur Urol. 2009;56(5):786-93. 